\title{
Design of a new intelligent temperature-controlling fan
}

\author{
Wu Tiebin ${ }^{1, a}$, Liu Yunlian ${ }^{1, b^{*}}$, Zhao Qiaomei ${ }^{1, c}$, Li Xinjun ${ }^{1, d}$,He Hang ${ }^{1, e}$ \\ ${ }^{1}$ Hunan University of Humanities, Science and Technology, Loudi, Hunan 41700, China \\ awutiebin81@163.com, bliuyunlian85@163.com, czqm80@163.com, d31531084@qq.com \\ ehehang@qq.com
}

\begin{abstract}
Keywords: Electric fan ; Intelligent; Fuzzy expert rules; Environment temperature
Abstract. A new intelligent temperature-controlling, speed-regulating electric fan was designed. Environment temperature and human body surface temperature were separately detected by using a temperature sensor DS18B20 and a non-contact measuring transducer for surface temperature. On this basis, fuzzy expert rules comprehensively considering environment humidity and temperature as well as human body temperature were designed. Then according to the designed fuzzy expert rules, the speed of the fan was set. The fan which is humanized and energy-saving has a wide application prospect.
\end{abstract}

\section{Introduction}

People often use some equipment relating temperature in life. For example, although numerous urban families install air-conditioning systems, rural areas where live a majority of population in China still use electric fans to cool and prevent from sunstroke. As it turns from spring to summer or from summer to autumn, it is still hot in daytime ${ }^{[1-2]}$, and people lower the temperature by using electric fans with a high wind speed and large air flow. At night, temperature drops, so the wind speed of fans should be gradually declined to avoid people getting a cold after falling asleep. Although the wind speed of electric fans can be adjusted to different grades, people need to shift wind speed manually. While, this cannot be realized after people falling asleep. For this problem, people usually turn off electric fans by timing in advance ${ }^{[3-4]}$. However, first, the timing period limited, within 1-2 hours in general. Second, temperature possibly not declines sufficiently after one or two hours, but fans have been closed according to the regulated time. Thus, people are probably awakened up because of high temperature, and then have to get up to turn on electric fans again and lengthen the timing period, which are quite troublesome. What's worse, if the last timing period is too long to turn off fans after temperature drop, people are likely to have a cold ${ }^{[5]}$. Third, electric fans only have a single function of turning off at the regulated time but fail to meet different requirements of temperature changes for wind speed of fans.

With technology progress, continuous economic development and improved living standard, people have increasingly high requirements for household appliances which are required to be more healthy, energy-saving, safe, intelligent and humanized. Under such context, traditional electric appliances gradually show their shortcomings and electric fans, as one of household appliances, also have similar problems. For example, the temperature of the first half of the night is high, so the fan is set to generate wind at a high speed. However, the temperature drops at the second half of the night while the wind speed cannot decrease with the decrease of the temperature after people fall asleep so that people, especially children, are easily to have a cold. The primary reason of these shortcomings is the lack of detections for the environment temperature and the body surface temperature of human. In order to solve the above problems, a set of environment-controlled auto-fan system is designed, with characteristics including automation, intelligence, environmental protection and humanization.

\section{Design framework of the intelligent fan based on environment and human body temperatures}

The new intelligent temperature-controlling and speed-regulating electric fan includes a single-chip microcomputer, a reset circuit, temperature and humidity gathering circuits, a circuit for driving nixie tubes, a nixie tube, a power regulating circuit, a fan, a secondary single-chip microcomputer and a 
speed measurement device. The hardware structure is shown in Figure 1. The humidity gathering circuit includes a sensor for collecting environment temperature and a non-contact measuring transducer for collecting surface temperature of human bodies. The two sensors are separately connected with the single-chip microcomputer through signal lines. The single-chip microcomputer is connected with the fan via the power regulating circuit and electrolysis. In addition, the power regulating circuit includes an optoelectronic isolator and a control circuit for controllable silicon. The working process of the intelligent fan is as follow: Environment temperature and human body surface temperature are separately detected by using the temperature sensor DS18B20 and the TS118-3 non-contact measuring transducer for surface temperature (infrared detection). The detected signals are continually sent to the 89C51 single-chip microcomputer, which calculate the rotation speed of the fan according to the expert rules after comprehensively considering temperature and humidity. Then the rotation speed is turned into a control quantity and driven as a command to make the motor rotate according to the set procedure. Afterwards, the Hall integrated sensor feeds back the detected rotation speed of the motor to the single-chip microcomputer, which then makes judgments according to the feedback signals. The motor is re-driven according to the initial value set for the single-chip microcomputer. Through repeated adjustments like this, the electric fan can stably work at the rotation speed set for corresponding temperatures. As a result, the rotation of the electric fan is adjusted through detecting the external environments.

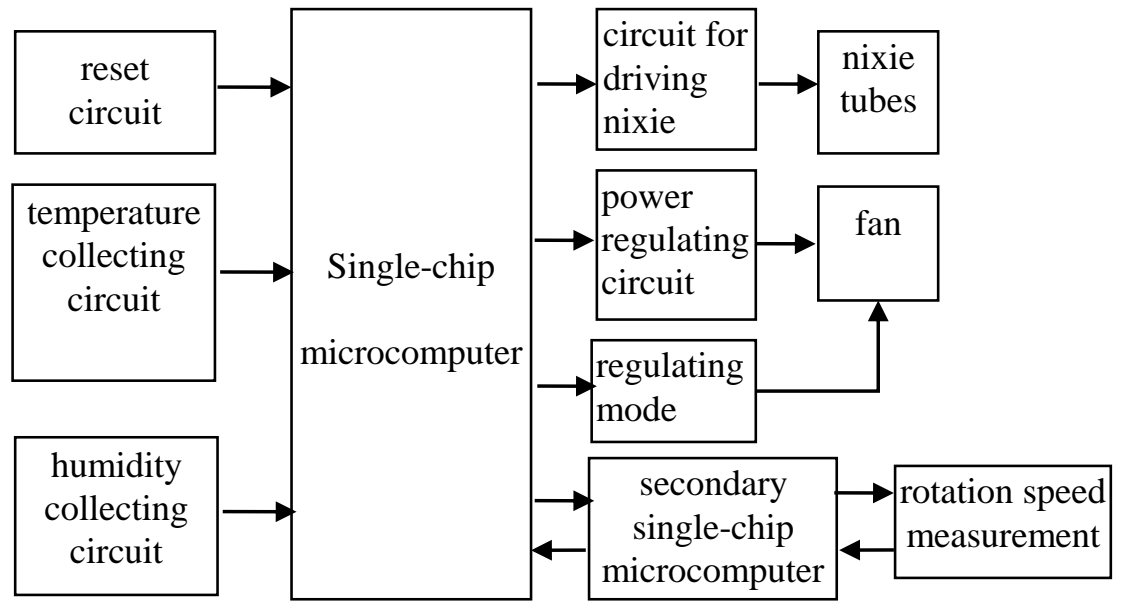

Figure 1 Control structure of the intelligent fan

\section{Hardware design of the intelligent speed-regulating fan}

\section{Main control chip (single-chip microcomputer)}

The STC89C51 single-chip microcomputer, as the core of the control system, is an eight-bit complementary metal oxide semiconductor (CMOS8) single-chip microcomputer with low voltage and high performance. It supports online programming and basically meets the demand of programming. In addition, it has advantages including high integration, small volume, high reliability, strong control function, low power consumption and voltage as well as high cost performance.

\section{Temperature collecting circuit}

After being detected using the TS118-3 non-contact measuring transducer for surface temperature, the infrared rays radiated from human are transmitted to the A/D 7705 transverter to be magnified and converted to figure signals and sent to the single-chip microcomputer. Then the single-chip microcomputer samples, quantizes and codes the figure signals and transmit them into the nixie tube for display. The display time of the nixie tube and the human temperature range can be adjusted by using the keyboard circuit 


\section{Humidity collecting circuit}

HS1101 capacitive sensor is equivalent to a capacitor in the circuit structure and its capacitance increases with the increase of measured air humidity. To precisely convert the capacitance variations into computer-readable singles easily, the HS1101 capacitive sensor is placed into the 555 oscillating circuit first to convert the changes of capacitance into inversely proportional voltage frequency signals. Finally, the detected period is shown on the oscilloscope and the humidity is calculated using relevant formula.

\section{Power regulating circuit}

The power controlling part includes the optoelectronic isolator and the control circuit for controllable silicon. The optoelectronic isolator isolates strong current signals from weak ones and eliminates signal interference of noise signals and common ground clutters in the input circuit on the output circuit so as to ensure safe and reliable operation of the system. The maximum working voltage and current of the optoelectronic isolator MOC3021 are $240 \mathrm{~V}$ and $15 \mathrm{~mA}$, respectively. The delay pulse output from P1 interface line of the single-chip microcomputer is sent to the control level of the bidirectionally controllable silicon BTA12 through the optoelectronic isolator so as to change the output power of the electrical fan.

\section{Rotation speed measurement}

The small Hall integrated sensor A44E merely with three pins is applied. One pin is connected with VCC, another pin is connected with GND, and the rest one is the output end. The Hall integrated sensor A44E is fixed on the protecting net of the electrical fan and a magnetic sheet is pasted on the fan blades facing the sensor. Because of Hall effects, when the magnetic sheet passes by the Hall sensor with the rotation of the fan blade, the output end of the Hall sensor produces a pulse. The output pulse is sent to the TO end of the single-chip microcomputer for count, and the number of pulses per unit time means the rotation speed of the electrical fan. Another 89C51 is employed in the external to enable the secondary single-chip microcomputer to process frequency measurement data and constantly sent the frequency words to the host 89C51 through the serial port. Some simple functions can also be completed by the secondary $89 \mathrm{C} 51$.

\section{Software designs of the intelligent speed-regulating fan}

\section{Speed-regulating mode based on pulse-width modulation}

The rotation speed of the motor can be controlled by through pulse-width modulation (PWM) of the single-chip microcomputer. PWM based speed regulation is achieved by the software programming of the single-chip microcomputer. PWM is a modulation mode for adjusting output quantities and wave shape by changing the width of pulse sequences according to certain rules. In the regulating system of PWM driving control, rectangular wave PWM signals are most commonly used and the duty ratio of PWM waves needs to be regulated in the modulation. Duty ratio refers to the percentage of duration time of high level within a periodic time. When controlling the rotation speed of the motor, the higher the duty ratio is, the faster the rotation speed. If the duty ratio reaches to $100 \%$, that is, all the PWM waves are at high level, the fan is rotated at the maximum speed.

\section{Setting of wind speed based on fuzzy expert rules}

T-environ and T-skin represent environment temperature and human body temperature, respectively. $\mathrm{H}$-humid refers to the humidity of environment, and S-wind represents wind speed. Because the temperature, humidity and wind speed are all fuzzy concepts, they cannot be solved using traditional expert rules. However, fuzzy expert rules can favorably solve these cases because they adopt fuzzy technologies in the process of acquiring and representing knowledge. 
The fuzzy sets of environment temperature, human body surface temperature and environment humidity are divided into seven language variables. The fuzzy subsets are $\{\mathrm{ZO}, \mathrm{PS}, \mathrm{PM}, \mathrm{PB}\}$, respectively, in which the elements ZO, PS, PM and PB represent zero, positive small, positive medium and positive big, respectively. Then a fuzzy knowledge base is established and a fuzzy inference machine is determined. The inference forms of three typical fuzzy expert rules with variable universe are listed as follows:

(1) If $\mathrm{T}$-environ=PB, T-skin= $\mathrm{PB}$ and $\mathrm{H}$-humid $=\mathrm{PB}$, then $\mathrm{S}$-wind=PB. That is to say, when environment and human body surface are at high temperatures along with high environment humidity, it needs to set the wind speed at the maximum value to cool down as soon as possible.

(2) If $\mathrm{T}$-environ $=\mathrm{NB}, \mathrm{T}$-skin $=\mathrm{NB}$ and $\mathrm{H}$-humid $=\mathrm{NB}$, then $\mathrm{S}$-wind $=\mathrm{ZO}$. It means that when temperatures of environment and human body surface as well as environment humidity are low, the wind speed has to be set as zero. It is unnecessary to cool down, or people may have a cold.

(3) If T-environ=PB, T-skin=PS and H-humid=PS, then $\mathrm{S}$-wind=PM. It means that when the wind speed is set at a medium level if temperature of environment is high while human body temperature and environment humidity are low. It is unnecessary to lower the temperature, or people may have a cold.

\section{Conclusion}

The intelligent fan designed in this study applying integrated temperature sensor with high precision and controlled by the single-chip microcomputer is easy to be operated and can show real-time temperature. Moreover, the intelligent fan automatically generates winds at low and high speeds and turns off at the pre-set temperatures with high precision. According to the detected indoor temperature, human body surface temperature and environment humidity, the rotation speed of the motor in the electrical fan is automatically changed to realize various functions, including automatic switch of different wind speeds, self turn-on according to pre-set temperatures and turning off at low temperatures. The research verifies that the intelligent fan has a promising application prospect as it meets people's demands for products with health, safety, energy conservation and emission reduction as well as environmental protection characteristics.

\section{Acknowledgements}

This work was partially supported by the Loudi science and technology project(NO.16), Scientific Research Fund of Hunan Provincial Education Department(NO.14B097, NO.15C0722), and Natural Science Foundation of Hunan Province(2016JJ3079), and the Youth Project of Hunan university of Humanities, Science and Technology (2009QN04)

Corresponding author: :Liu yunlian, E-mail: liu yunlian85@163.com

\section{References}

[1] Xia Zebin; Zhang Guangping; Xie Yonghong. Air conditioning fan control system based on single chip microcomputer. China Science and technology information, 2011,100-101

[2] Han Xingguo; Su Qingyong; Wang Qing. Smart fan control system design based on STC89C52 MCU. The equipment manufacturing technology, 2013,53 55

[3] Liang Guoxiang. Hardware design of fan intelligent controller based on single chip microcomputer. Journal of Ningde Normal University (natural science edition), 2012,173-175

[4] Xie Zhiping. Intelligent temperature control fan based on single chip microcomputer. New technology and new products in China, 2011,152-153

[5] Wang Rui. PWM motor speed control system based on AT89S52 single chip microcomputer. Journal of Yan'an Vocational and Technical College, 2012, 120-121 